\title{
Elaboración y validación de un documento informativo sobre adeno-amigdalectomía para pacientes
}

\author{
Elaboration and validation of an informative document \\ on adenoamigdalectomy for patients
}

\author{
M.J. Escudero-Carreteroํㅜ, S. Sánchez-Gómez ${ }^{2}$, R. González-Pérez ${ }^{1}$, R. Sanz-Amores ${ }^{3}$, \\ M.A. Prieto-Rodríguez ${ }^{1}$, E. Fernández de la Mota ${ }^{3}$
}

\section{RESUMEN}

Fundamento. El acceso a una información sanitaria actualizada y comprensible es una demanda ciudadana creciente, así como un deber ético y una norma legal. La información por escrito refuerza la información que se transmite verbalmente en la consulta y mejora la capacidad de recuerdo. La adenoamigdalectomía es una intervención que afecta a un número elevado de pacientes, mayoritariamente a una población muy sensible emocionalmente, la infantil, cuyos padres y madres lo viven con bastante ansiedad. Se elabora y valida una guía informativa sobre adeno-amigdalectomía. El objetivo de este trabajo es describir el proceso de elaboración del documento, destacando el procedimiento de validación del mismo y sus resultados.

Material y métodos. Validación del documento en tres fases: A) de contenidos clínicos por profesionales con checklist y reunión de consenso. B) análisis de legibilidad gramatical según el índice de Flesch. C) validación de legibilidad léxica con ciudadanía a través de guía de valoración y entrevista.

Resultados. La valoración de profesionales y ciudadanía fue muy positiva. Profesionales destacaron la exactitud de los contenidos y corrigieron algunas imprecisiones. Hicieron sugerencias respecto a formato, redacción y extensión. Ciudadanía destacó: lenguaje comprensible, resolución de dudas, tono respetuoso y satisfacción por esta iniciativa. Difieren también en aspectos como la consideración sobre la extensión.

Conclusiones. La validación de documentos informativos tanto con profesionales como con pacientes es un proceso de mucho interés. Al no coincidir en sus análisis se pone de manifiesto la importancia de incluir a ambos sectores. El documento mejoró con las aportaciones.

Palabras clave. Información. Comunicación en salud. Educación en salud. Folleto informativo para pacientes.

\begin{abstract}
Background. Access to up-to-date and understandable health information is a growing citizen demand, as well as an ethical duty and a legal regulation. Written information reinforces information transmitted orally during the consultation and improves recall capacity. Adenoamigdalectomy is an intervention that affects a high number of patients, the majority from the child population that has a high emotional sensitivity, and it is experienced by parents with considerable anxiety. An informative guide on adenoamigdalectomy was elaborated and validated. The aim of this article is to describe the process of elaborating the document, emphasizing its validation and results.
\end{abstract}

Material and methods. The document was validated in three phases: A) Clinical content validated by professionals with a checklist and a consensus meeting; B) Analysis of grammatical legibility according to the Flesch index; C) Validation of lexical legibility with citizens by means of a validation guide and interviews.

Results. The validation by professionals and citizens was highly positive. The professionals stressed the accuracy of the content and corrected some imprecisions. They made suggestions concerning format, writing style and length. The citizens stressed understandable language, resolution of doubts, respectful tone and their satisfaction with the initiative. They also differed on aspects such as their opinion of the document's length.

Conclusions. The validation of informative documents by both professionals and patients is a process of great interest. Lack of agreement in their analyses makes it clear why it is important to include both sectors. The document was improved by their contributions.

Key words. Information. Health communication. Health education. Informative pamphlet for patients.
1. Escuela Andaluza de Salud Pública. Granada 2. Hospital Universitario Virgen Macarena. Sevilla 3. Dirección General de Calidad, Investigación y Gestión del Conocimiento. Consejería de Salud y Bienestar Social. Junta de Andalucía. Sevilla

Recepción: 19 de octubre de 2012

Aceptación provisional: 21 de noviembre de 2012

Aceptación definitiva: 21 de diciembre de 2012

\section{Correspondencia}

María J. Escudero Carretero

Escuela Andaluza de Salud Pública

Granada

E-mail: maria.escudero.easp@juntadeandalucia.es

Financiación: La realización del proyecto que da origen a este artículo fue financiada por la Dirección General de Calidad, Investigación y Gestión del conocimiento de la Consejería de Salud (Junta de Andalucía, España). La publicación de este artículo no supone conflicto de intereses. 


\section{INTRODUCCIÓN}

La información es una herramienta básica en los procesos individuales de enfermedad-salud y es un requisito ético imprescindible para ejercer el derecho a la autonomía: decidir en función de los propios valores y preferencias ${ }^{1-2}$. Este principio ético es recogido y desarrollado en nuestro ordenamiento jurídico por varias leyes, desde la Ley General de Sanidad de 1986 que enuncia la primera carta de derechos y deberes del paciente y, con ella establece la norma sobre el consentimiento informado, hasta la Ley Básica de Autonomía del Paciente, entre otras ${ }^{3-5}$.

Por otra parte, el acceso a una información sanitaria suficiente, clara y precisa es una demanda cada vez más extendida entre la ciudadanía. Familiares y pacientes expresan la necesidad de manejar más información sobre su proceso de salud, sobre el diagnóstico, el tratamiento, el pronóstico y los cuidados. Quieren información accesible y fácil de entender, actualizada, adecuada a sus circunstancias y necesidades y que contemple los aspectos emocionales de la enfermedad ${ }^{6-15}$.

Acceder a una información completa y de calidad sobre el propio proceso de salud es preciso para poder tomar decisiones sobre el mismo, decisiones acordes con los propios valores y preferencias; lo que incrementa la efectividad de las terapias, garantiza un derecho sanitario de la ciudadanía y mejora su satisfacción ${ }^{8-10,16-17}$. No tener información suficiente y fiable es uno de los factores que ocasionan más conflicto en el proceso de toma de decisiones sobre la propia salud ${ }^{11}$.

No obstante, la información no siempre se transmite de la manera más idónea. Con frecuencia, la información se traslada sólo de forma verbal durante el acto clínico y ligada a la comunicación de un diagnóstico o de un pronóstico, cuando las personas están menos receptivas y más preocupadas. Otras veces, se ofrece demasiada información, sin adaptarla ni cuidar las implicaciones emocionales. Por otra parte, la información que seleccionan y ofrecen los profesionales no siempre es suficiente o es la que quieren o necesitan sus pacientes ${ }^{14-15,18}$.
La información transmitida sólo verbalmente, con frecuencia, da lugar a malentendidos $\mathrm{u}$ olvidos ${ }^{19}$. La información por escrito es una herramienta muy eficaz para compensar estas deficiencias, ya que presenta numerosas ventajas. La retención y comprensión de la información mejora significativamente si la información oral se acompaña de información por escrito $^{10,19-20}$; reduce la ansiedad y mejora el uso de medidas preventivas y de autocuidado, así como la adherencia al tratamiento. La información por escrito previene problemas de comunicación entre profesionales sanitarios y pacientes, reforzando el rol profesional y aumentando la confianza de sus pacientes. Este tipo de información, además, favorece un uso más adecuado y efectivo de los servicios de salud ${ }^{10,16,18-20}$.

La información por escrito se puede retomar siempre que se necesite. Constituye un apoyo para el futuro y es un instrumento que ayuda a garantizar la accesibilidad a una información correcta ${ }^{16}$. Además, la ciudadanía demanda que la información sea accesible a través de distintos canales, específicamente, quieren información por escrito que complemente la información profesional $^{12}$. En un estudio realizado en Canadá, el $75 \%$ de la población encuestada expresó su deseo de recibir materiales informativos por escrito ${ }^{11}$. Un estudio llevado a cabo en Andalucía señala cómo las personas quieren recibir información a través de otros medios que complementen la recibida por el personal sanitario. En concreto, sobre la información escrita, las personas que, en ocasiones anteriores, han recibido la información en este soporte, están muy satisfechas con él y desean seguir recibiendo información por este medio ${ }^{15}$.

Sobre la elaboración de materiales informativos escritos para pacientes y cuidadoras existe un cuerpo de conocimiento bastante extenso que ha dado lugar a distintas guías y recomendaciones. Así, ya sabemos que la información ha de estar basada en la mejor evidencia científica disponible, lo que supone un trabajo previo de revisión de la literatura científica y una actualización de la misma y de los materiales que se elaboren ${ }^{8,10,20}$. La información debe 
estar expresada de forma clara y comprensible, utilizando palabras sencillas y sin tecnicismos ${ }^{20-25}$, aclarando dudas, no creándolas, desmontando creencias erróneas y expresada en un tono respetuoso e incluyente y no paternalista ni alarmista ${ }^{8,21}$.

La adenoamigdalectomía es una intervención que afecta a un número elevado de pacientes, suponiendo un importante volumen de la práctica quirúrgica en un hospital. Afecta mayoritariamente a una población muy sensible emocionalmente, la infantil ${ }^{26}$. Sus conocidos riesgos e importantes complicaciones han sido objeto preferente de los documentos informativos para pacientes, sobre todo de los consentimientos informados. Contrasta la baja frecuencia de aparición de estas complicaciones con las necesidades informativas de pacientes y sus familiares, que superan las ofrecidas por los consentimientos informados. Por esta razón, desde la Consejería de Salud del gobierno andaluz y, en el contexto de la definición e implementación del Proceso Asistencial Integrado Adeno-Amigdalectomía, se planteó la necesidad de elaborar un documento con información adi- cional para pacientes sobre dicho proceso asistencial. El objetivo de este artículo es describir el proceso de elaboración del documento, destacando el procedimiento de validación del mismo y sus resultados.

\section{MATERIAL Y MÉTODOS}

Para elaborar el documento informativo sobre adeno-amigdalectomía se elaboró una metodología que consta de siete fases (Fig. 1). Dichas fases se pueden agrupar en dos momentos: 1) elaboración del documento y 2) revisión y validación del mismo. Todo este procedimiento está sustentado y orientado en una serie de criterios de calidad definidos por el equipo a partir de las recomendaciones más habituales para la elaboración de información para pacientes (Tabla 1). En este artículo se describe fundamentalmente la segunda fase, el procedimiento de revisión y validación del contenido, por ser el objetivo central de este artículo. Dicho procedimiento responde a un diseño fundamentalmente cualitativo y fue realizado en Andalucía, en las provincias de Sevilla, Granada y Almería.

\begin{tabular}{|l|l|l|}
\hline Fase & \multicolumn{1}{|c|}{ Qué se hace } & \multicolumn{1}{|c|}{ Quién lo hace } \\
\hline $\mathbf{1}$ & \multicolumn{1}{|c|}{ Elaboración del documento } \\
\hline 1.1 & $\begin{array}{l}\text { Revisión evidencia científica. Búsqueda de otros } \\
\text { documentos informativos sobre adeno-amigdalec- } \\
\text { tomía para pacientes/cuidadoras. Revisión reco- } \\
\text { mendaciones sobre elaboración documentos escri- } \\
\text { tos para pacientes. }\end{array}$ & Equipo del proyecto \\
\hline 1.2 & $\begin{array}{l}\text { Elaboración de criterios de calidad a cumplir por } \\
\text { el documento. }\end{array}$ & Equipo del proyecto \\
\hline 1.3 & $\begin{array}{l}\text { Elaboración primer documento } \\
\text { Enálisis lenguaje para que no resulte sexista ni dis- }\end{array}$ & $\begin{array}{l}\text { Profesional con conocimiento sobre la patología (ORL) } \\
\text { y profesional de la comunicación con pacientes. }\end{array}$ \\
\hline 1.4 & $\begin{array}{l}\text { Equipo del proyecto: profesionales del equipo con } \\
\text { formación en estudios de género y experiencia en } \\
\text { transformación de textos al lenguaje no sexista. }\end{array}$ \\
\hline 2. & \multicolumn{2}{|c|}{ Revisión y validación del contenido } \\
\hline 2.1. & $\begin{array}{l}\text { Validación / Revisión de contenidos clínicos con } \\
\text { profesionales }\end{array}$ & $\begin{array}{l}\text { Participan: Grupo profesionales sanitarios. Modera- } \\
\text { ción coordinadora del equipo. }\end{array}$ \\
\hline 2.2. & $\begin{array}{l}\text { Análisis legibilidad gramatical del documento, si es } \\
\text { Validación / Revisión del documento con familia- }\end{array}$ & $\begin{array}{l}\text { Una profesional sanitaria experta en legibilidad de } \\
\text { textos informativos dirigidos a pacientes. }\end{array}$ \\
\hline
\end{tabular}

Figura 1. Fases en la metodología de la elaboración de un documento informativo para pacientes. 
Tabla 1. Criterios de calidad para la elaboración de documentos escritos con información adicional para pacientes

1. Implicación de pacientes y profesionales en el proceso de desarrollo de la información.

2. Definición inicial del propósito de la información, su objetivo y el público a quien va dirigida.

3. La información clínica estará basada en evidencia científica y en una revisión sistemática de la misma.

4. Con la información se dará respuesta a las preocupaciones o dudas más habituales de pacientes.

5. Utilización de un tono no alarmista ni paternalista, sino constructivo, positivo, que anime.

6. La información se transmitirá de una forma clara, fácil de comprender, sin tecnicismos, de la forma más breve posible y a través de caracteres muy legibles.

7. Se evitará duplicar informaciones.

8. Inclusión de información sobre los riesgos y beneficios de la intervención o tratamiento, si procede.

9. Se explicitará la autoría del documento y quién lo promueve y respalda.

10. Se explicitará la fecha de publicación y/o actualización.

11. Se incluirán referencias de las fuentes de evidencia o fuentes de referencia para posibles consultas.

12. Definición del momento del proceso sanitario en el que se facilitará el documento.

\section{Validación de contenidos clínicos por profesionales}

Con el objetivo de garantizar que los contenidos clínicos del documento informativo sobre adeno-amigdalectomía fueran veraces, completos y actualizados, se invitó a un grupo de profesionales sanitarios a validar el documento. En la constitución del grupo se buscó representatividad de todos los perfiles profesionales que participan en la atención sanitaria a la adeno-amigdalectomía: especialistas en otorrinolaringología, pediatría, medicina de familia, anestesia y enfermería. Se optó por buscar un número de personas suficiente para poder trabajar con él y llegar a consensos, siguiendo las recomendaciones realizadas para grupos de trabajo con metodologías cualitativas, entre 8 y 12 personas $^{27}$.

La dinámica de trabajo fue la siguiente:

1. Se realizó una reunión de presentación del proyecto donde se explicó qué tipo de documento se pretendía elaborar, los criterios de calidad determinados y el procedimiento metodológico. Se distribuyó el documento borrador y un checklist para guiar la valoración. Dicho checklist incluía un listado de las dimensiones claves a valorar en el documento, junto con los criterios de calidad determinados previamente para el mismo. Los aspectos a valorar incluidos fueron: estructura del documento, contenido del mismo, estilo, diseño e ilustraciones. Se pidió al grupo que revisara que los contenidos del documento inicial se ajustaban a la evidencia científica y que respondía a las dudas más frecuentes de pacientes y sus padres y madres. Dispusieron de quince días para ello.

2. Cada integrante del grupo realizó una valoración individual del documento.

3. Se hizo una reunión de consenso para poner en común las valoraciones individuales y llegar a acuerdos sobre los contenidos definitivos. 


\section{Análisis de legibilidad gramatical}

Según Aliende (citado en Barrio y col), la legibilidad "es el conjunto de características de los textos que favorecen o dificultan una comunicación más o menos eficaz entre ellos y los lectores, de acuerdo con la competencia de éstos y con las condiciones en que realizan la lectura" ${ }^{28}$. Un texto resulta más comprensible, cercano y ameno cuando cumple criterios de legibilidad, tanto lingüística como tipográfica. A su vez, la legibilidad lingüística se compone de la legibilidad gramatical (estructura y construcción gramatical) y la léxica (significado de las palabras $)^{28-29}$. Aunque en la elaboración del documento se cuidaron todos estos aspectos, en este punto de la valoración del mismo, se hizo un análisis de la legibilidad gramatical, para garantizar que se cumplía este criterio.

Existen diversos métodos para analizar la legibilidad gramatical de un texto, como la fórmula RES de Flesch, el SMOG, el Gráfico de Fry, la Fórmula de Perspicuidad de Szigrist, entre otras ${ }^{28-30}$. En este estudio, el nivel de legibilidad gramatical del documento se analizó mediante una herramienta específica y validada para evaluar la legibilidad de los textos en castellano dirigidos a pacientes: el programa informático INFLESZ (Escala INFLESZ), que se basa en el cálculo de la Fórmula de Perspicuidad (Índice de Flesch-Szigriszt) ${ }^{29}$.

Este índice es un indicador de la dificultad para leer un texto. Se obtiene de la razón de las palabras no comunes y las palabras comunes que aparecen en un texto basándose para ello en un vocabulario básico definido. Si se pretende que un texto resulte legible para la población media española dicho índice debe estar por encima de $55^{29}$

\section{Validación de legibilidad léxica con pacientes $\mathrm{y} / \mathrm{o}$ familiares}

Se consideró este paso imprescindible con el objetivo de comprobar: a) el grado de interés de los temas seleccionados para los pacientes y b) el grado de comprensión del texto. Para hacerlo se ideó un procedimiento cualitativo basado en tres pasos:
1. El equipo y los profesionales sanitarios implicados en el proyecto definieron los perfiles típicos de pacientes de adenoamigdalectomía y sus familiares o personas cuidadoras.

2. Dichos profesionales, en sus consultas, identificaron padres y madres de pacientes de adeno-amigdalectomía en base a esos perfiles, buscando la representatividad de todos los posibles discursos y la máxima heterogeneidad del grupo participante (en cuanto a características como edad, sexo, nivel de estudios, ocupación o procedencia geográfica).

3. Se informó a las personas seleccionadas sobre el propósito del proyecto.

4. Se les pidió por escrito su consentimiento para participar.

5. Se les proporcionó el documento informativo y una guía para la valoración que se llevaron y contestaron individualmente en el centro de salud o en sus domicilios. Esta guía de valoración (Tabla 2) fue elaborada ad hoc por el equipo del proyecto, al no encontrar ningún instrumento ya publicado y validado que sirviera para ello. Para desarrollarla se realizó una búsqueda exhaustiva de documentación sobre cómo elaborar documentos con información adicional para pacientes, se sintetizaron unos criterios de calidad que han de tener dichos documentos (y que deben tener los documentos elaborados en el marco de este proyecto) (Tabla 1) y se aislaron unas dimensiones básicas de análisis para este tipo de documentos. En base a esas dimensiones y criterios, se construyeron unas preguntas y se diseñó la guía que incluye algunas preguntas cerradas, pero la mayoría son abiertas.

6. De vuelta en la consulta, entregaron la guía cumplimentada y sostuvieron una breve entrevista semiestructurada con su profesional. 
Tabla 2. Guía para la valoración del documento informativo para pacientes sobre adeno-amigdalectomía.

\begin{tabular}{|c|c|c|}
\hline \multicolumn{3}{|l|}{ ¿Cuál es su primera impresión y su valoración global? } \\
\hline \multicolumn{3}{|l|}{$\begin{array}{l}\text { ¿Hay algo que llame especialmente su atención positiva o } \\
\text { negativamente? ¿Qué y porqué? }\end{array}$} \\
\hline ¿Queda claro el objetivo de la guía? ¿Cuál es? & $\begin{array}{l}\text { Sí } \\
\text { No }\end{array}$ & Indique por qué: \\
\hline $\begin{array}{l}\text { ¿Queda claro a quién va dirigida esta información? ¿A } \\
\text { quién? }\end{array}$ & $\begin{array}{l}\text { Sí } \\
\text { No }\end{array}$ & Indique por qué: \\
\hline $\begin{array}{l}\text { ¿Le parece que está escrita de una forma clara? ¿Se entiende } \\
\text { fácilmente? }\end{array}$ & $\begin{array}{l}\text { Sí } \\
\text { No }\end{array}$ & Indique por qué: \\
\hline ¿Hay algunas palabras cuyo significado no comprenda? & $\begin{array}{l}\text { Sí } \\
\text { No }\end{array}$ & Indique por qué: \\
\hline $\begin{array}{l}\text { ¿Utiliza un lenguaje alarmista en algún momento? ¿Hay algo } \\
\text { que le haya asustado? }\end{array}$ & $\begin{array}{l}\text { Sí } \\
\text { No }\end{array}$ & Indique por qué: \\
\hline ¿El tono es respetuoso? & $\begin{array}{l}\text { Sí } \\
\text { No }\end{array}$ & Indique por qué: \\
\hline ¿Es importante y necesaria la información que aporta? & $\begin{array}{l}\text { Sí } \\
\text { No }\end{array}$ & Indique por qué: \\
\hline ¿Dice suficiente sobre causas y consecuencias? & $\begin{array}{l}\text { Sí } \\
\text { No }\end{array}$ & Indique por qué: \\
\hline ¿Dice suficiente sobre riesgos y beneficios? & $\begin{array}{l}\text { Sí } \\
\text { No }\end{array}$ & Indique por qué: \\
\hline ¿La guía le aclara sobre el proceso sanitario que va a seguir? & $\begin{array}{l}\text { Sí } \\
\text { No }\end{array}$ & Indique por qué: \\
\hline ¿Le gusta el orden de presentación de los contenidos? & $\begin{array}{l}\text { Sí } \\
\text { No }\end{array}$ & Indique por qué: \\
\hline ¿Está toda la información que le interesa? & $\begin{array}{l}\text { Sí } \\
\text { No }\end{array}$ & Indique por qué: \\
\hline ¿Hay alguna información que eche en falta? & $\begin{array}{l}\text { Sí } \\
\text { No }\end{array}$ & Indique por qué: \\
\hline ¿Hay alguna información que le parezca que no es cierta? & $\begin{array}{l}\text { Sí } \\
\text { No }\end{array}$ & Indique por qué: \\
\hline $\begin{array}{l}\text { ¿Están recogidas en esta guía informativa todas sus preocu- } \\
\text { paciones? }\end{array}$ & $\begin{array}{l}\text { Sí } \\
\text { No }\end{array}$ & Indique por qué: \\
\hline $\begin{array}{l}\text { ¿Le parece útil el epígrafe final donde puede escribir? ¿Cree } \\
\text { que lo usará? }\end{array}$ & $\begin{array}{l}\text { Sí } \\
\text { No }\end{array}$ & Indique por qué: \\
\hline $\begin{array}{l}\text { ¿Qué profesional le parece que le debería entregar esta guía } \\
\text { informativa? }\end{array}$ & \multicolumn{2}{|c|}{$\begin{array}{l}\text { Algunas opciones, señale cuál: } \\
\text { - Médico de Familia } \\
\text { - Especialista en Otorrinolaringología. } \\
\text { - Anestesista } \\
\text { - Profesional de enfermería de Atención Primaria } \\
\text { - Profesional de enfermería de hospital } \\
\text { - Profesionales de admisión }\end{array}$} \\
\hline \multicolumn{3}{|l|}{ ¿Hay algo que quitaría de esta guía informativa? } \\
\hline \multicolumn{3}{|l|}{ ¿Hay algo qué añadiría? } \\
\hline ¿Cómo mejoraría este documento? & & \\
\hline
\end{tabular}




\section{RESULTADOS}

\section{Valoración de contenidos clínicos por profesionales sanitarios}

Participaron doce profesionales que habitualmente trabajan en el proceso asistencial de adeno-amigdalectomía: cinco especialistas en otorrinolaringología, cuatro especialistas en otras áreas de la medicina: pediatría, medicina de familia y anestesia y tres profesionales de enfermería.

Su valoración global fue excelente. Realizaron muchas sugerencias sobre el contenido, aunque también sobre aspectos no buscados a priori como la redacción, el formato o la extensión del documento.

Sobre el contenido, se valoró que se ajustaba perfectamente a la evidencia científica más actualizada. Tan sólo se señalaron algunas imprecisiones, como una cierta confusión existente en el documento entre riesgos y complicaciones. Se propuso también la inclusión de un índice de contenidos, del que carecía el primer documento.

De la redacción, se destacaron algunos fragmentos del texto cuya redacción les parecía algo alarmante. Y se puso de manifiesto la necesidad de acortar las frases, algunas de las cuales se calificaron de "largas".

En cuanto al formato, se comentó la pertinencia de sumar al escrito, la utilización de otros canales de transmisión de la información, haciendo especial hincapié en los audiovisuales. También se sugirió publicarlo en un tamaño fácilmente manejable. Y se consideró muy extenso el documento (tenía 13 páginas), llegando a afirmar un profesional que "nadie se va a leer un documento tan largo". Finalmente, el grupo valoró que merecía la pena incluir todos los contenidos abordados y valorar la extensión en la validación con pacientes.

Respecto al momento idóneo para entregar este documento informativo las y los profesionales opinaron mayoritariamente que era preferible "entregarla en el momento más cercano a la decisión sobre la intervención quirúrgica". No obstante, también hubo alguna persona que recomendó entregarla en la consulta de Atención Pri- maria "para que tengan información clara cuando lleguen al especialista".

\section{Análisis de legibilidad gramatical}

Como se explicó en el apartado de métodos, el análisis de legibilidad gramatical se realizó a través del programa INFLESZ, según el cual un texto es legible cuando puntúa por encima de 55.

El texto no pasó el test de legibilidad en el primer análisis, obtuvo una puntuación de 51'538. Fue necesario revisar el documento y modificar la redacción: acortando las frases y sustituyendo algunas palabras largas por otras más cortas, a través de sinónimos. De esta forma, el segundo análisis de legibilidad fue superado sin dificultad obteniendo mejor puntuación: 60 .

\section{Valoración del documento por madres y padres de pacientes}

En la validación con la ciudadanía participaron 46 personas, padres $(n=17)$ y madres $(n=29)$ de pacientes con indicación de adenoamigdalectomía, con una edad media de 34 años (edad mín 20, máx 53). Estas personas trabajaban mayoritariamente en puestos no cualificados o realizando las labores domésticas, aunque también había tres estudiantes y diez personas que no contestaron sobre su ocupación.

La valoración global del documento fue muy positiva. La gran mayoría opinó que la guía era buena o muy buena. Comentaron que este documento mejoraba la calidad de la información recibida en la consulta, tranquilizaba y aportaba una información que buscarían por otros medios, quizás menos fiables, de no obtenerla en el propio sistema sanitario. Añaden que se trataba de una guía muy completa, clara y sencilla: "Buena, es una guía que detalla e informa como debe de ser".

En relación con los contenidos, la gran mayoría de las personas consideraron que la información proporcionada era importante y necesaria, ya que "informa bastante bien y anticipa al proceso que se va a vivir". Todas las personas estaban de acuerdo en 
que la guía aclaraba el proceso sanitario de la adeno-amigdalectomía.

Se obtuvo una respuesta unánime considerando suficiente la cantidad de información contenida en el documento sobre las causas y consecuencias de la intervención quirúrgica, así como sobre los riesgos y beneficios que conllevaba. No obstante, alguna persona comentó que "nunca es suficiente". El orden de los contenidos presentados en la guía es calificado positivamente "va por pasos y muy bien ordenaditos".

También fue mayoritario el número de personas que respondió que el documento recogía todas sus preocupaciones y dudas. Algunas personas solicitaron más información sobre:

1. El proceso concreto de la intervención quirúrgica

2. Más detalle sobre el postoperatorio:
a. Actividades diarias que se pueden realizar
b. Consecuencias de no tener ade- noides
c. Papel de la familia en esta etapa

3 . Nombres de medicamentos que necesitarán

4. Opiniones de pacientes que ya han pasado por el proceso

En cuanto a la legibilidad léxica o comprensibilidad, todas las personas pensaron que la guía estaba escrita de forma clara y fácil de comprender. Aún así, un 10\% de participantes manifiestaron no conocer el significado de todas las palabras que aparecían en el texto. Las palabras cuyo significado no se conocía eran "Adenoide" y "Disección".

En relación al lenguaje utilizado, todas las personas coincidían en que el texto mostraba un tono respetuoso y la mayoría pensaba que el lenguaje no era alarmista ni paternalista. No obstante, hay quien opinó que era un poco paternalista, así como alguna persona que expresaba que algunos de los términos utilizados asustaban.

El aspecto tipográfico no era el definitivo cuando se pilotó, por lo que no se pi- dió opinión sobre el mismo. Sin embargo, espontáneamente, recibió muchas sugerencias de mejora. Se prefería una guía con un tamaño más manejable (estaba impresa en tamaño DINA4: 21 x $30 \mathrm{cms}$ ), así como la inclusión de dibujos que amenizaran la lectura y ayudaran a comprender la intervención. Además, se invitaba a utilizar medios audiovisuales para la elaboración de futuros materiales y su difusión.

Más de la mitad de las personas entrevistadas mostraron su satisfacción con la inclusión al final del documento de un apartado reservado para anotaciones personales "es muy útil, porque puedes anotar cosas de interés que vayas a olvidar o cosas que sólo sean útiles para ti y no para otras niñas". Una persona recomendó que este epígrafe se encuentre en una hoja aparte de manera que se pudiera separar si fuera preciso.

La satisfacción con el documento fue generalizada entre las personas consultadas y felicitaron a la institución sanitaria andaluza por esta iniciativa.

Respecto al procedimiento de acceso a la guía, la mayoría de las personas consideraban que debería entregarla el especialista en otorrinolaringología al inicio del subproceso quirúrgico, cuando se indica la conveniencia de la intervención quirúrgica. Hubo personas que opinaron que la guía debería ofrecérsela su pediatra al comienzo del proceso, incluso si después no se establecía indicación quirúrgica. También sugerían que estuviera disponible en el servicio de admisión del hospital y en las consultas de los centros sanitarios (tanto hospitales como centros de salud), así como en Internet.

En la tabla 3 se sintetizan los resultados de las valoraciones de profesionales y padres y madres de pacientes. Por otro lado, algunos de los resultados obtenidos en las entrevistas con padres y madres de pacientes se han cuantificado (a partir de las respuestas a la guía de valoración por escrito). Los resultados se muestran en la tabla 4. 
Tabla 3. Valoración de la guía por profesionales, padres y madres de pacientes

\begin{tabular}{ll}
\hline \multicolumn{1}{c}{ Profesionales } & \multicolumn{1}{c}{ Padres y madres de pacientes } \\
\hline Valoración global & \\
\hline Excelente & $\begin{array}{l}\text { Buena o muy buena } \\
\text { Guía completa, clara y sencilla }\end{array}$ \\
\hline Valoración del contenido & \\
\hline $\begin{array}{l}\text { Aclaración de algún concepto confuso } \\
\text { Incluir una tabla de contenidos }\end{array}$ & $\begin{array}{l}\text { Aclara el proceso que se va a vivir } \\
\text { Se valora el orden de los contenidos }\end{array}$ \\
\hline Valoración de la redacción & $\begin{array}{l}\text { Recoge todas sus preocupaciones y dudas } \\
\text { Solicitan más información sobre algunos temas }\end{array}$ \\
\hline $\begin{array}{l}\text { Modificación fragmentos alarmantes } \\
\text { Acortar frases }\end{array}$ & $\begin{array}{l}\text { Algunos términos asustan } \\
\text { Redacción clara y fácil de comprender }\end{array}$ \\
\hline Valoración del formato & \\
\hline $\begin{array}{l}\text { Seguir con el documento por escrito } \\
\text { Complementar con medios de comunicación }\end{array}$ & $\begin{array}{l}\text { Incluir dibujos } \\
\text { audiovisual }\end{array}$ \\
Tamaño del documento escrito más manejable & Tamaño del documento escrito más manejable \\
\hline Momento idóneo para entregar guía & \\
\hline $\begin{array}{l}\text { Momento cercano a la decisión sobre la } \\
\text { intervención quirúrgica }\end{array}$ & Especialista ante la indicación del proceso \\
En la consulta de atención primaria & En la consulta de atención primaria \\
\hline
\end{tabular}

Tabla 4. Valoración cuantificada del documento por ciudadanía (padres y madres de pacientes)

\begin{tabular}{|c|c|c|c|}
\hline & & & \\
\hline & Sí & No & $\mathrm{NS} / \mathrm{NC}$ \\
\hline $\begin{array}{l}\text { ¿La información contenida en el documento es } \\
\text { importante y necesaria? }\end{array}$ & $0,847+/-0,05$ & $0,043+/-0,03$ & $0,108+/-0,045$ \\
\hline ¿Dice suficiente sobre causas y consecuencias? & $0,978+/-0,021$ & $0^{*}$ & $0,021+/-0,021$ \\
\hline ¿Dice suficiente sobre riesgos y beneficios? & $1^{*}$ & $0^{*}$ & $0^{*}$ \\
\hline ¿Le aclara el proceso sanitario de atención? & $1^{*}$ & $0^{*}$ & $0^{*}$ \\
\hline ¿Le gusta el orden de presentación de contenidos? & $0,913+/-0,041$ & & \\
\hline ¿Está toda la información que le interesa? & $0,913+/-0,041$ & $0,021+/-0,041$ & $0,065+/-0,036$ \\
\hline ¿Hay información que eche en falta? & $0,043+/-0,03$ & $0,0847+/-0,052$ & $0,108+/-0,045$ \\
\hline ¿Hay información que le parezca que no es cierta? & 0 & $0,934+/-0,036$ & $0,065+/-0,036$ \\
\hline ¿Está escrita de forma clara? & $1^{*}$ & $0^{*}$ & $0^{*}$ \\
\hline ¿Se utiliza un lenguaje alarmista o lee algo que le asuste? & $0,086+/-0,041$ & $0,782+/-0,06$ & $0,130+/-0,049$ \\
\hline ¿Está escrita en un tono respetuoso? & $1^{*}$ & $0^{*}$ & $0 *$ \\
\hline ¿Se utilizan palabras cuyo significado no conoce? & $0,108+/-0,045$ & $0,891+/-0,045$ & $0^{*}$ \\
\hline ¿Están recogidas todas sus preocupaciones? & $0,891+/-0,045$ & $0,043+/-0,03$ & $0,065+/-0,036$ \\
\hline
\end{tabular}

$\mathrm{n}=46$. IC $95 \%$.

No se puede calcular el IC.

Hay que tener en cuenta a la hora de leer estos valores que se ha realizado un muestreo cualitativo buscando representatividad de los distintos discursos que hay en la población y no un muestreo estadístico de la población. 
El documento de información sobre adeno-amigdalectomía está disponible en la siguiente dirección de internet: http:// www.csalud.juntaandalucia.es/salud/sites/ csalud/contenidos/Informacion_General/ c_6_c_7_guias_informacion_pacientes/ guia_adeno_amigdalectomia

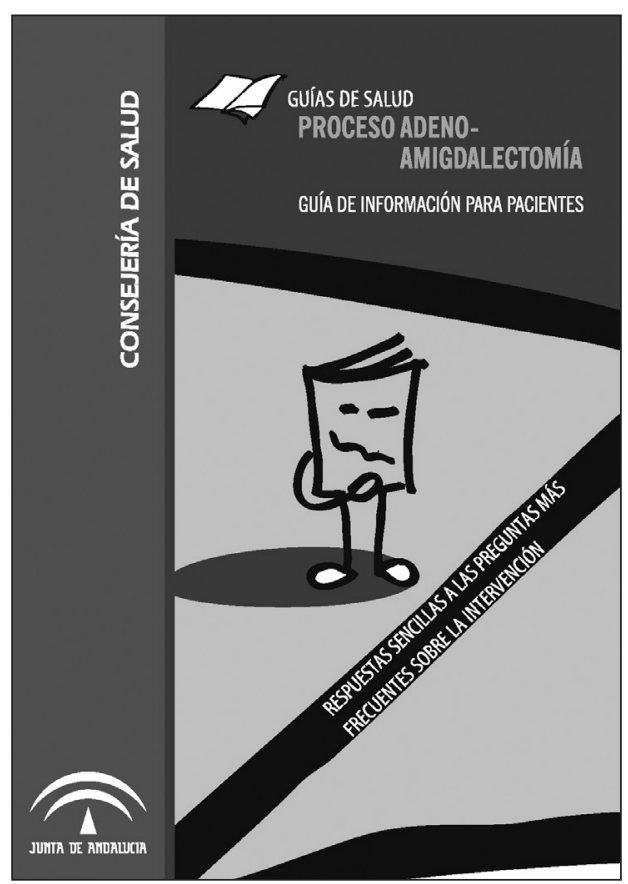

\section{DISCUSIÓN}

El documento informativo sobre adenoamigdalectomía fue elaborado siguiendo un procedimiento riguroso y cuidado. El resultado es una guía informativa que responde a los criterios de calidad previamente definidos: basada en la mejor evidencia científica disponible, con información suficiente sobre el proceso, fácilmente comprensible y que aclara la mayor parte de las dudas de la ciudadanía sobre la adenoamigdalectomía.

La valoración que realizan profesionales y familiares del documento es excelente, aunque enfatizan distintos aspectos. Los profesionales valoraron favorablemente los contenidos por su exactitud y alcance.
Los padres de los pacientes diagnosticados de adenoamigdalectomía destacaron la importancia de contar con información por escrito, afirmando que les había tranquilizado leer la guía y que habían afrontado mejor todo el proceso pre y post operatorio. Disponer de una información adecuada desde el inicio facilita el afrontamiento positivo de la enfermedad. La información sobre salud debe contribuir a disminuir la ansiedad, contrarrestar miedos y desmontar creencias erróneas ${ }^{31}$. Se confirman en nuestro ámbito los resultados de Bellew $\mathrm{y} \mathrm{col}^{32}$, estudio en el que se afirma que los progenitores de niñas y niños que van a ser intervenidos quirúrgicamente muestran altos niveles de ansiedad. Esta ansiedad la pueden trasladar a sus hijos y puede interferir en el proceso de recuperación, por lo que se destaca la importancia de prepararles psicológicamente para la intervención. Una forma de hacerlo es proporcionarles información por escrito que contribuye a rebajar los niveles de ansiedad ${ }^{32-34}$. En el estudio de Winterton y col, los pacientes participantes afirmaban que el folleto había mejorado su comprensión del procedimiento y que sabían mejor qué atención sanitaria podían esperar ${ }^{35}$. Los padres entrevistadas en nuestro trabajo afirman que hubieran buscado esta información por sí mismos, si sus profesionales sanitarios no se la hubieran proporcionado aunque ésta, elaborada por la institución sanitaria autonómica, les parece mucho más fiable.

Tanto profesionales como padres destacan también la claridad de los mensajes transmitidos en el documento, cumpliendo con una recomendación ampliamente extendida.

Es curioso comprobar la importancia que todas las personas que validaron el documento le dieron al formato. Aunque el texto fue pilotado y validado antes de incorporarle el diseño definitivo y no se les preguntó por el aspecto del documento, de forma espontánea, tanto profesionales como padres y madres hicieron diversas sugerencias de mejora sobre el mismo. Los profesionales señalaron la necesidad de incluir un índice del que carecía el documento. Este resultado coincide con la recomen- 
dación de facilitar que quienes consulten un documento encuentren fácilmente la información que buscan ${ }^{12}$ y que ésta esté bien organizada y estructurada ${ }^{24-25}$. Los padres solicitaron la inclusión de ilustraciones. Efectivamente, las figuras (fotos o dibujos) y pictogramas que refuercen los mensajes escritos ayudan a comprender y retener los mensajes y mejoran la legibilidad de los textos $^{36}$. Según Lagassé y col, el aspecto del documento es fundamental porque puede contribuir a mejorar su accesibilidad ${ }^{25}$. Y si es más accesible será más útil.

Otro resultado muy interesante se relaciona con la extensión del documento. Algunos de los profesionales opinaron que era demasiado largo y los pacientes no lo leerían entero. Sin embargo, los padres valoraron que la extensión era adecuada porque toda la información contenida era pertinente y contribuía a resolver sus dudas. Por esta razón no lo encontraron largo. Una persona realmente preocupada por un tema de salud está muy motivada para leer información relacionada con el mismo sin importarle excesivamente su extensión. Se constata la disociación entre las necesidades de información sobre salud de la ciudadanía y las teorías que sobre las mismas desarrollan los profesionales. Este resultado confirma la importancia de incluir en la elaboración de este tipo de documentos tanto a profesionales como a pacientes y/o representantes de la ciudadanía ${ }^{6,8,20,31}$.

La incorporación de profesionales de distintos ámbitos y especialidades en la elaboración y validación del documento fue muy positiva porque enriqueció el texto, que ganó en pluralidad y rigor. Así mismo, los resultados del pilotaje muestran la importancia de implicar a pacientes en la elaboración de la información porque supuso un refuerzo, la respuesta a algunas dudas del equipo e implicó la necesidad de aclarar aún más parte de la terminología empleada. Se considera altamente conveniente implicar a pacientes, como mínimo, en dos momentos del proceso de elaboración de documentos informativos: en el inicio, para conocer cuáles son sus necesidades informativas $\mathrm{y}$, al final, para evaluar el documento y comprobar si responde a esas necesidades y si es suficientemente comprensivo y claro $^{22,24,37}$. En este caso, sólo se hizo en el momento final y ésta es una de las limitaciones que presenta la metodología. De hecho, algunas personas indicaron otros temas sobre los que les gustaría tener más información, como se ve en los resultados. Otra cuestión es si era pertinente tratar o no esos temas en un documento de este tipo.

El documento se validó con los padres de los pacientes porque, en realidad, por su formato y terminología, está más dirigido a personas adultas. No obstante, siendo un proceso asistencial que afecta de forma relevante a pacientes pediátricos, se apunta la necesidad de elaborar un documento dirigido específicamente al público infantil y en un formato más adecuado, como el cómic o el cuento.

Una limitación de este estudio puede estar relacionada con la guía de valoración que se utilizó para los padres de los pacientes plasmaran por escrito su opinión sobre el documento. Al ser un instrumento que se realizó expresamente para este estudio y que no estaba validado anteriormente con un procedimiento riguroso, como el descrito en los artículos de Carvajal y col y Morilla-Herrera y $\mathrm{col}^{38-39}$, se puede afirmar que los resultados obtenidos con el mismo son descriptivos y orientativos, pero no generalizables.

La información juega un papel fundamental en el afrontamiento de cualquier proceso de enfermedad-salud y en la correspondiente toma de decisiones, facilitando que los pacientes adopten un rol más activo $^{11,36,40}$. Las organizaciones sanitarias y los equipos profesionales tienen la responsabilidad de asegurarse de que pacientes y familiares disponen de información suficiente sobre sus procesos de enfermedad y que han comprendido dicha información ${ }^{16}$. Los pacientes no deberían tener que buscar información por su cuenta ya que no todos y no siempre están en posición de hacerlo ${ }^{12}$. Proporcionar información por escrito mejora la comprensión y recuerdo de la información, pero no sustituye la necesaria interacción con el/a médico/a a $^{1631,37}$. Aunque se deberían seguir mejorando las 
habilidades de comunicación de los y las profesionales sanitarios $^{9-10}$, se verifican los óptimos resultados que se consiguen con la elaboración de materiales por escrito.

\section{Agradecimientos}

Queremos agradecer la colaboración en este proyecto del grupo de profesionales sanitarios del SSPA que han contribuido con sus conocimientos y su tiempo a la realización de este documento informativo, ya sea validando sus contenidos o facilitando el acceso a sus pacientes y haciendo la entrevista para la validación del documento. Así mismo, agradecemos a todas las personas, madres y padres de pacientes, que han participado en la validación del documento, aportando su opinión y sugerencias.

\section{BIBLIOGRAFÍA}

1. GRACia D, Júdez J (EDS). Ética en la práctica clínica. Madrid: editorial Triacastela. 2004.

2. Ferrer JJ, Álvarez JC. Para fundamentar la bioética. Madrid: Universidad Pontificia de Comillas/DDB. 2003.

3. Simón Lorda P, Barrio CANTAlejo IM. Un marco histórico para una nueva disciplina: la bioética. Med Clin 1995; 105: 583-597.

4. Ley $14 / 1986$, de 25 de abril, General de Sanidad, publicada en el BOE nº 102 de 29 de abril de 1986 .

5. Ley 41/2002, de 14 de noviembre, Básica reguladora de la Autonomía del paciente y de derechos y obligaciones en materia de información y documentación clínica, publicada en BOE nº 274 de 15 de noviembre de 2002.

6. Moumuid N, Morelle M, Carrère MO, Bachelot T, MignotTe H, BRÉMOND A. Elaborating patient information with patients themselves: lessons from a cancer treatment focus group. Health Expect 2003; 6: 128-139.

7. Prieto Rodríguez MA, Suess A, Escudero CarreTERo MJ, I FERNÁNDEZ FERnÁNDEZ, JC MARCH CERDA. Las expectativas de los/as pacientes en 12 procesos asistenciales del sistema sanitario público de Andalucía. En: Consejería de Salud. Premios de Investigación en Salud 2004. Sevilla, Consejería de Salud 2005; 47-75.

8. Coulter A, Entwistle V, Gilbert D. Sharing decisions with patients: is the information good enough? BMJ 1999; 318: 318-322.
9. Schattner A, Bronstein A, Jeluin N. Information and shared decision-making are top patients' priorities. BMC Health Services Research 2006; 6: 21-26.

10. ARThur VAM. Written patient information: a review of the literature. Adv Nurs 1995; 21: 1081-1086.

11. O'Connor AM, Drake ER, Wells GA, Tugwell P, Laupacis A, Elmslie T. A survey of the decisionmaking needs of Canadians faced with complex health decisions. Health Expect 2003; 6: 97-109.

12. NICKLING J. Improving the quality of written information for patients. Nurs Stand 2002; 16 : 39-44.

13. Jovell AJ. El paciente del siglo XXI. An Sist Sanit Navar 2006; 29 (Supl. 3): 85-90.

14. Semere W, Karamanoukian H L, Levitt M, Edwards T, Murero M, D’Ancora G et al. A pediatric surgery study: parent usage of the internet for medical information. Pediatri Surg 2003; 38: 560-564.

15. González Pérez R, Gijón Sánchez MT, Escudero Carretero MJ, Prieto Rodriguez MA, Carles March J, Ruiz Azarola A. Perspectivas de la ciudadanía sobre necesidades y expectativas de información sanitaria. Rev Calidad Asistencial 2008; 23: 101-108.

16. PALMerini J, Jasovsky DA. Patient Education: a guide for success. Nursing Management 1998; 29: 45-46.

17. Delgado A, López-Fernández LA, de Dios-Luna J, Saletti-Cuesta L, Gil-Garrido N, Puga-GonzÁlez A. Expectativas de los pacientes sobre la toma de decisiones ante diferentes problemas de salud Gac Sanit 2010; 24: 66-71.

18. Vlastos IM, Hajiloannou J, Houlakis M. Otitis media with effusion: what parents want to know. J Laryngol Otol 2008; 122: 21-24.

19. Chan Y, Irish JC, Wood SJ, Rotstein LE, Brown DH, Gullane PJ et al. Patient Education and informed consent in head and neck surgery. Arch Otolaryngol Head Neck Surg. 2002; 128 : 1269-1274.

20. Moult B, Franck LS, Brady H. Ensuring Quality Information for Patients: development and preliminary validation of a new instrument to improve the quality of written health care information. Health Expect 2004; 7: 165-175.

21. HAS: Haute AuTorité de SANTÉ. Guide méthodologique. Élaboration d'un document écrit d'information à l'intention des patients et des usagers du système de santé. París: HAS; 2005. (acceso 15 de marzo de 2010). Disponible en: http://www.has-sante.fr/portail/ jcms/c_430286/how-to-produce-an-information-brochure-for-patients-and-healthcaresystem-users 
22. DoAk CC, DoAk LG, Root JH. Teaching patients with low literacy skills. $2^{\text {a }}$ ed. Philadelphia: J.B. Lippincott Company; 1996.

23. Hill-Briggs F, Schumann K, Dike O. Five-step methodology for evaluation and adaptation of print patient health information to meet the $<5$ th grade readability criterion. Medical Care 2012; 50: 294-301.

24. Barrio IM, Simón-Lorda P, Melguizo M, Molina A. Consenso sobre los criterios de legibilidad de los folletos de educación para la salud. An Sist Sanit Navar 2011; 34: 153-165.

25. Lagassé LP, Rimal RN, Smith KC, Storey JD, RhoADEs E, BarnetT DJ et al. How accesible was information about H1N1 Flu? Literacy assesments of CDC guidance documents for different audiences. PloS ONE 2011; 6: e23583.

26. Sánchez-Gómez S, Abrante a, Caballero MM, CeBallos L, Cordero JM, Fernádez A et al. Proceso asistencial integrado Amigdalectomía/Adenoidectomía. Sevilla: Consejería de Salud; 2003.

27. Alfonso-SÁnchez JL. Técnicas de planificación sanitaria para la creatividad (III). Cap 7 pp: 127-35. En Alfonso-Sánchez JL. Servicios de Salud. Técnicas avanzadas de marketing y planificación sanitaria. Madrid: Editorial Doyma. 1991.

28. Barrio Cantalejo IM, Simón Lorda P. Medición de la legibilidad de textos escritos. Correlación entre método manual de Flesch y métodos informáticos. Aten Primaria 2003; 31: 104-108.

29. Barrio-Cantalejo im, Simón-Lorda P, Melguizo M, Escalona I, MariJuán MI, HeRnando P. Validación de la Escla INFLESZ para evaluar la legibilidad de los textos dirigidos a pacientes. An Sist Sanit Navar 2008; 31: 135-152.

30. IdoAtE VM. La comprensibilidad del consentimiento informado. An Sist Sanit Navar 2000; 23:109-113.

31. Domínguez-Nogueira C, Expósito-Hernández J, García-Wiedemann EJ. Análisis de la calidad de los documentos informativos destinados a pacientes con cáncer. Rev Calidad Asistencial 2005; 20: 377-384.

32. Bellew M, Atkinson KR, Dixon G, Yates A. The introduction of a paediatric anaesthesia information leaflet: an audit of its impact on parental anxiety and satisfaction. Paediatr Anaesth 2002; 12: 124-130.

33. Sово EJ. Parent's perceptions of pediatric day surgery risks: unforeseeable complications, or avoidable mistakes? Soc Sci Med 2005; 60: 2341-2350.

34. Alonso-López J. Programa de preparación psicológica en cirugía infantil programada. Revista profesional española de terapia cognitivo-conductual 2005; 3: 56-70.

35. Winterton RiS, Alaani A, Loke D, Bem C. Role of information leaflets in improving the practice of informed consent for patients undergoing septoplasty. J Laryngol Otol 2007; 121 : 134-137.

36. MANSOor LE, Dowse R. Effect of pictograms on readability of patient information materials. Ann Pharmacother 2003; 37: 1003-1009.

37. Kubba H. An evidence-based patient information leaflet about otitis media with effusion. Clin Perform Qual Health Care 2000; 8: 93-99.

38. Carvajal A, Centeno C, Watson R, Martínez M, SAnz-Rubiales A. ¿Cómo validar un instrumento de medida de la salud? An Sist Sanit Navar 2011; 34: 63-72.

39. Morilla-Herrera JC, Morales-Asencio JM, FernándeZ-Gallego MC, Berrobianco Cobos E, DelgaDo Romero A. Utilidad y validez de un instrumento basado en indicadores de la Nursing Outcomes Classification como ayuda al diagnóstico de pacientes crónicos de Atención Primaria con gestión ineficiente de la salud propia. An Sist Sanit Navar 2011; 34: 51-61.

40. JACKson C, ChEATER FM, Reis I. A systematic review of decision support needs of parents making child decisions. Health expectations 2008; 11: 232-251. 
